\title{
Development of Dairy Cattle Registration and Herd Management System
}

\author{
Hongchao $\mathrm{Wu}^{1,2}$, Xibo Qiao ${ }^{1}$, Xin $\operatorname{Luan}^{1}$, Biao $\mathrm{Li}^{1}$, Zhongle Chang ${ }^{1}$, \\ Jinghe Tan ${ }^{1}$, and Xinzhong Fan ${ }^{1, *}$ \\ ${ }^{1}$ College of Animal Science \& Technology, Shandong Agricultural University, \\ Taian, P.R. China, 271018 \\ sdfxz@163.com \\ ${ }^{2}$ Shandong University of Traditional Chinese \\ Medicine Jinan, P.R. China, 250355
}

\begin{abstract}
In order to meet the requirement of dairy cattle breeding and modern cattle farm management, the dairy cattle registration and herd management system was programmed with Visual FoxPro9.0, which can run on Windows9X/Me/NT/2000/XP, to fit the current implemented Canadian dairy cattle DHI recorders and 9-point linear comprehensive evaluation. Based on data collection and analysis of basic herd information and individual information on milk production, reproductive performance, body type score, health status, feeding and progeny performance, the system can be used for herd management, cow evaluation and breed registration, intelligent mating selection and suggestion for improving farm management. The application in several different scale farms shows it can improve the efficiency of farms management and cattle breeding significantly.
\end{abstract}

Keyword: Dairy cattle, Registration, Management software, Visual FoxPro.

\section{Introduction}

Modern breeding and production of dairy cattle is an open system with a long span time, a lot affecting factors and much complex structure, which depends on the application of accurate and systematic data management and computer software technology. Compared with the North American and European countries, China dairy cattle breeding started later and had poor foundation. The organization management was distemperedness, and software used in cattle registration and herd management was less. The collection and management of breeding and production data were relied on manual in small dairy farms, which was not only consuming time no guaranteeing the normative of data, but also inconveniencing statistical analysis. With the rapid development of China dairy husbandry recently, the scale and level of dairy farms have been continuously improved. New dairy cattle breeding and production

\footnotetext{
* Corresponding author.
} 
management system is necessary to meet the demand of Dairy Cattle Breeding program for the extension of Canadian dairy cattle DHI and 9-point linear comprehensive evaluation.

\section{System Design}

\subsection{System Design Object}

Referencing the existing hardware and software foundation in our country, the system was programmed with Visual FoxPro9.0, combining with modern dairy cattle production and management techniques and the requirement of dairy cattle breeding.

According to the modularization method of "I-P-D", relational database technology and theory of structural life cycle method were applied to ensure the rationality, integrity and security of data structure and the stable operation of the system. It has many functions such as collection of dairy cattle breeding and production data, dairy cattle registration, daily management, semen management and assisted matching. By using the system, technical personnel can determine the direction of dairy cattle improvement according to the analysis of performance and body type, arrange daily tasks and make production report in view of the dairy production rhythm, and do auxiliary production management in dairy cattle farms.

\subsection{System Module Design}

Module structure was divided into three layers: control module, Sub-control module and functional module. Every module was related to key management content of dairy cattle breeding. System module structure is shown in Figure 1.

\section{Function of System}

\subsection{Password and Permission}

Each operator will have own password and permission, which is effective to prevent illegal and ultra vires operation, and make authorization more impartial and authoritative.

\subsection{Data Input, Validation and Transformation}

The information of production management in cattle farm includes the data of propagation, milking production, the dry period and cattle registration, etc. In view of the statistical analysis of the information, technical personnel can discover and solve problems in production in time. For some relatively fixed data or information, users can easily select them with mouse. The system can automatic check the data inputted by users. When the data is unreasonable it can give warning and prompt timely. Meanwhile, the system can provide a data interface to invoke data with other formats, modify and transform them promptly. 


\subsection{Image Acquisition, Call and Management}

It is a difficult problem to easily manage images for non-software professional. The system applies a series of procedures to identify different kinds of images, automatic search images with the same prefix name based on cattle numbers and display the card in the cattle file accurately. Operators only need to get digital photos of cows, turn the prefix name to the corresponding cattle number, and then transfer them to the system's picture folder. The management of images becomes simple and effective.

\subsection{Body Type Identification and Results Show}

According to 9-point linear comprehensive evaluation of Canada, technical personnel can input the data of 24 traits and 39 common defects of 5 parts about dairy cattle. Integral and partial score are calculated shown by columnar diagram with standard deviation from average, which is very direct and visual, meeting the international popular practice.

\subsection{Data Query, Collection and Output}

Users can timely change the information according to the change situation of dairy cattle and achieve a combination of inquiry in variety of conditions. Query results can be directed to the printer or the other format files. SQL statements and query optimization technique of RUSHMORE were applied to make the query speed fast as far as possible.

\subsection{Notice of Daily Management Tasks}

Daily management and determination of dairy cattle is a very complex matter, because the management of dairy cattle has long cycle and massive data. The system can forecast dry period, calving time and the cows need to be measured and improve dairy management efficiency.

\subsection{Management of Transference, Mobilization and Elimination}

In daily management, transference, mobilization and elimination of dairy cattle are very universal. In order to facilitate the daily management, the system provides of mobility management module, which is close to the reality, and manages kinds of data rationally.

\subsection{Semen Managing and Assisted Matching}

The system has set up the module of bull/semen registration. Technical personnel can input all the bull pedigree, photos, body conformation, production performance, breeding value, etc., and achieve the information of all bulls so as to choose the most appropriate one to mate cow. The module of assisted matching can provide a list of unrelated bulls automatically in view of cow pedigree and performance characteristics. 


\subsection{Maintenance and Help}

The software not only has initialization and running environment settings, but also applies two database mechanisms: transaction processing and data cache. Log disposal technology was used, in order to ensure the security and stable operation of the system. A detailed manual was prepared for operators providing the using method of each module and some knowledge of dairy cattle breeding.

\section{Popularization and Application}

At present, the system has been installed and popularized in ten dairy cattle farms and farming communities in shandong province, such as the first and second dairy cattle breeding farms of Jinan Jiabao, the Tianyuan dairy cattle farm in Mingshui, etc. It has been proven that this software has many advantages: (1) Owing to the succinct interface and the simple operation, people with little computer foundation can operate it easily. (2) The operation of data input and output is very convenient, and the data can be transformed among different formats. (3) The installation, maintenance and upgrade of the system are convenient. (4) The running of the system is fast and stable, and takes up less system resources. In a word, the system can meet the requirements of dairy cattle registration, breeding and production and improve the management efficiency. However, in our country, the application of dairy cattle breeding management software still belongs to the starting stage. The management of most dairy cattle farms is not standardized. The production arrangement in various farms is not the same, and might change with the enhancement of technical level. In order to adapt to the requirements of modern dairy cattle breeding and production management, we should study the internationally advanced procedure and strengthen the interaction with users to enhance the function of software.

\section{References}

1. Sun, C., Han, Z.: Chinese version programming foundation and demonstration of Visual FoxPro6.0. Electronic Industry Press, Beijing (2001)

2. Wang, G., Han, Z., et al.: Development of a software for data management on dairy farm. Animal Husbandry and Veterinary Medicine 35(9), 15-16 (2003)

3. Li, Y.: Design of computer management system on dairy farm. China Dairy Cattle (4), 14 (1999) 